\title{
AXISYMMETRIC BUCKLING OF A SPHERICAL SHELL EMBEDDED IN AN ELASTIC MEDIUM UNDER UNIAXIAL STRESS AT INFINITY
}

\author{
by G. W. JONES ${ }^{\ddagger}$, S. J. CHAPMAN and D. J. ALLWRIGHT \\ (OCIAM, Mathematical Institute, University of Oxford, \\ 24-29 St Giles', Oxford, OX1 3LB, England)
}

[Received XX Xxxx 200x. Revise XX Xxxx 200x]

\begin{abstract}
Summary
The problem of a thin spherical linearly-elastic shell, perfectly bonded to an infinite linearly-elastic medium is considered. A constant axisymmetric stress field is applied at infinity in the matrix, and the displacement and stress fields in the shell and matrix are evaluated by means of harmonic potential functions. In order to examine the stability of this solution, the buckling problem of a shell which experiences this deformation is considered. Using Koiter's nonlinear shallow shell theory, restricting buckling patterns to those which are axisymmetric, and using the Rayleigh-Ritz method by expanding the buckling patterns in an infinite series of Legendre functions, an eigenvalue problem for the coefficients in the infinite series is determined. This system is truncated and solved numerically in order to analyse the behaviour of the shell as it undergoes buckling, and to identify the critical buckling stress in two cases, namely where the shell is hollow and the stress at infinity is either uniaxial or radial.
\end{abstract}

\section{Introduction}

In underwater applications, anechoic tiles are often used to minimise the acoustic reflection coefficient of structures submerged in water. These tiles may consist of a rubber substrate containing a significant number of microscopic $(\sim 20 \mu \mathrm{m}$ radius $)$ hollow elastic spheres, whose thickness is around $2 \%$ of their radius. The pressure of the water causes the tiles to be compressed uniaxially, eventually causing the shells to buckle, thus softening the material (1). A first step in understanding this behaviour is an analysis of the buckling of a single embedded spherical shell.

The theory of shell buckling has a long and distinguished history. Euler's eighteenthcentury model for the elastic stability of a thin beam laid the groundwork for further studies into the buckling of plates and shells, which remains to this day an area of great research interest. Significantly for our investigation, the interaction of a shell with an elastic substrate or matrix affects its buckling behaviour considerably. Much work has been carried out into the buckling of cylindrical shells embedded in a linearly-elastic medium $(\mathbf{2}, \mathbf{3}, \mathbf{4}, \mathbf{5})$. More recently, work on composite materials containing carbon nanotubes has reignited interest in this field $(\mathbf{6}, \mathbf{7})$. However, away from cylindrical geometries, research is less abundant. Modelling the effect of a surrounding medium by a nonlinearly elastic foundation, Luo and Teng (8) considered a number of axisymmetric shells and their buckling under certain loads.

\footnotetext{
$\ddagger$ Corresponding author (jonesg@maths.ox.ac.uk)
}

Q. Jl Mech. Appl. Math. (200x) xx (x), 1-21

(C) Oxford University Press 200x 
Fok and Allwright (9) examined the buckling of embedded complete spherical shells, with a radial stress field applied at infinity in the substrate. However this analysis, as we shall see, involves a simplifying assumption which renders it invalid in many parameter regimes.

Our investigation will follow the spirit of Koiter's analysis of the buckling of a complete spherical shell under external pressure (10), which employs shallow shell theory. Following a physical description of the problem in Section 2 we review the necessary elements of buckling theory in Section 3. The state of stress in the shell prior to buckling is determined in Section 4 using a method presented by Love (11). The buckling problem is then solved in Sections 5 and 6 using the Rayleigh-Ritz method, which yields an infinite system of linear equations for coefficients of the basis functions in the Rayleigh-Ritz expansion. This system is solved numerically by truncation and the results are discussed in Sections 7 and 8 .

Throughout we restrict ourselves to axisymmetric displacements, which greatly simplifies the analysis. This means that we determine only when an axisymmetric solution bifurcates from the unbuckled solution, which will give us an upper bound for the critical load. We believe that for shells under compression (which is our primary interest) the first bifurcation will indeed be to an axisymmetric state; however, it is likely that shells under tension may first buckle to a nonaxisymmetric state.

A number of the investigations into the buckling of shells attached to elastic substrates (cited above) model the substrate as a Winkler foundation, essentially replacing the matrix by a bed of springs (either linear or nonlinear). The chief drawback of this model is that the effect of the matrix can only be felt locally; i.e. the response of the matrix to a localised shell deformation does not affect the matrix outside that region of deformation. Various models have been proposed to include non-local effects in the matrix while keeping the simplicity of the Winkler model (notably the Pasternak model (12)) but we choose to apply the more realistic model of a matrix, infinite in extent, which satisfies the equations of linear elasticity. This assumption is valid provided the strains in the matrix do not become too large. We will show in Section 7 that the applied stress at infinity for which buckling occurs is low enough that the matrix remains in the linear regime. Were this not the case, we would still hope that the results obtained could be useful as a qualitative description of the buckling process.

\section{Physical description of the problem}

The configuration of the problem is shown in Figure 1. We consider a spherical shell with internal radius $R_{0}$ and external radius $R_{1}$ embedded in an isotropic linearly-elastic matrix. By setting $R_{0}=\widehat{R}-h / 2$ and $R_{1}=\widehat{R}+h / 2$, we can alternatively say that the shell has a spherical mid-surface of radius $\widehat{R}$ and a constant thickness $h$. The matrix is characterised by its shear modulus $G_{\mathrm{m}}$ and Poisson ratio $\nu_{\mathrm{m}}$, and likewise the shell is characterised by $G_{\mathrm{s}}$ and $\nu_{\mathrm{s}}$.

We assume that the shell is perfectly bonded to the matrix, so that the displacement and traction at $R=R_{1}$ are continuous. In addition, we impose that the state of stress in the shell is a superposition of two states: the response to a uniaxial stress $\left.\tau_{z z}\right|_{\infty}=-q_{z}$ at infinity, and the (purely radial) response to the applied stresses $\left.\tau_{R R}\right|_{\infty}=-q_{R}$ and $\left.\tau_{R R}\right|_{R_{0}}=-q_{\text {in }}$.

We adopt the convention that vectors and tensors indexed with numeric subscripts and superscripts are the covariant and contravariant components respectively. Of these 


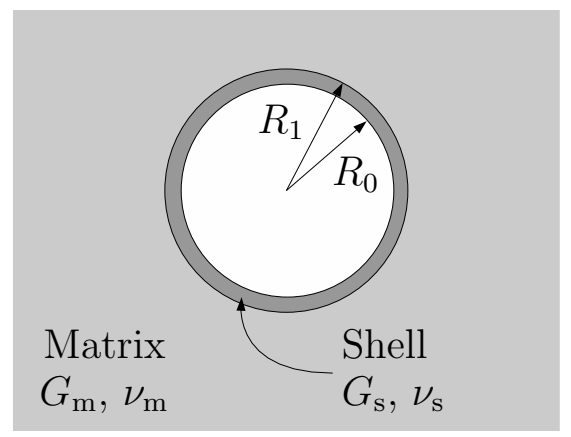

Fig. 1 Configuration of the physical problem.

indices, Greek letters vary over 1,2 and denote surface quantities; for the spherical polar coordinate system that we use in this article, ' 1 ' corresponds to the (colatitudinal) $\theta$ direction and ' 2 ' to the (azimuthal) $\phi$-direction. Latin letters vary over $1,2,3$ and denote three-dimensional quantities. In the spherical polar case the corresponding directions are $R, \theta, \phi$ respectively. Vectors and tensors indexed with a coordinate (in our article, $x, y, z$ for Cartesian components and $R, \theta, \phi$ for spherical polar components) such as $\tau_{R R}$ above, are referred to unit vectors, so that these are the physical components of the relevant vector or tensor.

\section{Buckling criterion}

In a stable equilibrium configuration the potential energy $W$ is a local minimum, so is stationary with respect to arbitrary infinitesimal virtual displacements $\boldsymbol{v}$. At a bifurcation point, corresponding to the emergence of a buckled solution, the equilibrium switches from being a minimum to a saddle point, and the quadratic (in $\boldsymbol{v}$ ) terms in $W$ are also stationary with respect to a particular virtual displacement $\boldsymbol{v}_{\mathrm{c}}$ (the buckling displacement). This condition is sometimes known as the Trefftz criterion.

We now consider how this theory applies to the shell embedded in a matrix. Firstly, in our analysis the virtual displacement $\boldsymbol{v}$ will refer to the virtual shell displacement; the virtual displacement in the matrix will be found as a linear function of $\boldsymbol{v}$, as the matrix obeys linear elasticity. The total potential energy of the system will be given by the sum of the potential energy in the shell and the potential energy in the matrix. We will first consider the shell. The derivation of these equations can be found, for instance, in Niordson (13); the notation, however, is that of Koiter (10).

The potential energy density of a thin shell can be written

$$
V=\frac{1}{2} h E^{\alpha \beta \lambda \mu} \gamma_{\alpha \beta} \gamma_{\lambda \mu}+\frac{1}{24} h^{3} E^{\alpha \beta \lambda \mu} \rho_{\alpha \beta} \rho_{\lambda \mu},
$$

where $\gamma_{\alpha \beta}$ is the middle-surface strain tensor, $\rho_{\alpha \beta}$ is the tensor of changes of curvature, and

$$
E^{\alpha \beta \lambda \mu}=G_{\mathrm{s}}\left(a^{\alpha \lambda} a^{\beta \mu}+a^{\alpha \mu} a^{\beta \lambda}+\frac{2 \nu_{\mathrm{s}}}{1-\nu_{\mathrm{s}}} a^{\alpha \beta} a^{\lambda \mu}\right)
$$

is the elasticity tensor for shells, where $a^{\alpha \beta}$ is the contravariant metric tensor of the shell's middle-surface. 
Denoting the pre-buckled state by the superscript (0), we suppose that the displacement in the shell is given by $\boldsymbol{v}^{(0)}+\boldsymbol{v}$, where $\boldsymbol{v}$ is the virtual displacement. Using the expression (3.1) for the potential energy density in the shell, the change in potential energy density, denoted $\Delta V$, is given by

$$
\begin{aligned}
\Delta V= & \frac{h}{2} E^{\alpha \beta \lambda \mu}\left(\gamma_{\alpha \beta}^{(0)}+\gamma_{\alpha \beta}\right)\left(\gamma_{\lambda \mu}^{(0)}+\gamma_{\lambda \mu}\right)+\frac{h^{3}}{24} E^{\alpha \beta \lambda \mu}\left(\rho_{\alpha \beta}^{(0)}+\rho_{\alpha \beta}\right)\left(\rho_{\lambda \mu}^{(0)}+\rho_{\lambda \mu}\right) \\
& \quad-\frac{h}{2} E^{\alpha \beta \lambda \mu} \gamma_{\alpha \beta}^{(0)} \gamma_{\lambda \mu}^{(0)}-\frac{h^{3}}{24} E^{\alpha \beta \lambda \mu} \rho_{\alpha \beta}^{(0)} \rho_{\lambda \mu}^{(0)} \\
= & \frac{h}{2} E^{\alpha \beta \lambda \mu}\left(\gamma_{\alpha \beta}^{(0)} \gamma_{\lambda \mu}+\gamma_{\alpha \beta} \gamma_{\lambda \mu}^{(0)}+\gamma_{\alpha \beta} \gamma_{\lambda \mu}\right)+\frac{h^{3}}{24} E^{\alpha \beta \lambda \mu}\left(\rho_{\alpha \beta}^{(0)} \rho_{\lambda \mu}+\rho_{\alpha \beta} \rho_{\lambda \mu}^{(0)}+\rho_{\alpha \beta} \rho_{\lambda \mu}\right) .
\end{aligned}
$$

Henceforth we suppose that the in-surface displacement components $v_{\alpha}$ of the shell are referred to the base vectors of the shell middle-surface, and that $w$ is the normal displacement, so that $\boldsymbol{v}=v_{\alpha} \boldsymbol{a}^{\alpha}+w \boldsymbol{a}^{3}$.

We will determine the pre-buckled state using the hypothesis of linear shell theory, so that terms which are of quadratic order or higher in the strain tensor are negligible. Thus we replace the pre-buckling middle-surface strain tensor, denoted $\gamma_{\alpha \beta}^{(0)}$, by its linearised counterpart $\theta_{\alpha \beta}^{(0)}$, which is defined by

$$
\theta_{\alpha \beta}=\frac{1}{2}\left(\left.v_{\alpha}\right|_{\beta}+\left.v_{\beta}\right|_{\alpha}\right)-b_{\alpha \beta} w
$$

where the notation $\left.v_{\alpha}\right|_{\beta}$ denotes covariant differentiation and $b_{\alpha \beta}$ is the second fundamental tensor of the surface. Furthermore, we replace the tensor of changes of curvature $\rho_{\alpha \beta}^{(0)}$ by the linear term $\bar{\rho}_{\alpha \beta}=\left.w\right|_{\alpha \beta}$. Additionally, by the principle of virtual work, we can relate the middle-surface strain tensor and the tensor of changes of curvature to the stress resultants $n^{\alpha \beta}$ and stress couples $m^{\alpha \beta}$ of the shell in the prebuckled state by

$$
{ }^{(0)} n^{\alpha \beta}=h E^{\alpha \beta \lambda \mu} \theta_{\lambda \mu}^{(0)}, \quad{ }^{(0)} m^{\alpha \beta}=\frac{h^{3}}{12} E^{\alpha \beta \lambda \mu} \bar{\rho}_{\lambda \mu}^{(0)} .
$$

Thus

$$
\Delta V={ }^{(0)} n^{\alpha \beta} \gamma_{\alpha \beta}+{ }^{(0)} m^{\alpha \beta} \rho_{\alpha \beta}+\frac{h}{2} E^{\alpha \beta \lambda \mu} \gamma_{\alpha \beta} \gamma_{\lambda \mu}+\frac{h^{3}}{24} E^{\alpha \beta \lambda \mu} \rho_{\alpha \beta} \rho_{\lambda \mu} .
$$

We now assume that the response of the shell to the virtual displacement satisfies the nonlinear shallow shell theory as employed by Koiter (10). In this theory, the strain measures are given by

$$
\gamma_{\alpha \beta}=\theta_{\alpha \beta}+\frac{1}{2} w_{, \alpha} w_{, \beta}, \quad \rho_{\alpha \beta}=\bar{\rho}_{\alpha \beta}=\left.w\right|_{\alpha \beta} .
$$

A nonlinear shell theory for the virtual displacements is essential for buckling problems, and the shallow shell approximation is the simplest nonlinear theory, containing just one nonlinear term. Thus we find the linear terms in $\Delta V$ are

$$
\Delta V_{1}={ }^{(0)} n^{\alpha \beta} \theta_{\alpha \beta}+{ }^{(0)} m^{\alpha \beta} \rho_{\alpha \beta}
$$


while the quadratic terms are

$$
\Delta V_{2}=\frac{1}{2}{ }^{(0)} n^{\alpha \beta} w_{, \alpha} w_{, \beta}+\frac{h}{2} E^{\alpha \beta \lambda \mu} \theta_{\alpha \beta} \theta_{\lambda \mu}+\frac{h^{3}}{24} E^{\alpha \beta \lambda \mu} \rho_{\alpha \beta} \rho_{\lambda \mu} .
$$

The reason for choosing the nonlinear theory for the virtual displacement is now apparent, since if we had not included the nonlinear term $\frac{1}{2} w_{, \alpha} w_{, \beta}$ in $\gamma_{\alpha \beta}$, then $\Delta V_{2}$ would be independent of the pre-buckled state.

The change in potential energy of the shell due to the virtual displacement is therefore

$$
\begin{aligned}
\Delta W_{\mathrm{s}}= & \iint_{\substack{\text { mid-shell } \\
\text { surface }}}\left({ }^{(0)} n^{\alpha \beta} \theta_{\alpha \beta}+{ }^{(0)} m^{\alpha \beta} \rho_{\alpha \beta}\right) \mathrm{d} S \\
& +\iint_{\substack{\text { mid-shell } \\
\text { surface }}}\left(\frac{1}{2}{ }^{(0)} n^{\alpha \beta} w_{, \alpha} w_{, \beta}+\frac{h}{2} E^{\alpha \beta \lambda \mu} \theta_{\alpha \beta} \theta_{\lambda \mu}+\frac{h^{3}}{24} E^{\alpha \beta \lambda \mu} \rho_{\alpha \beta} \rho_{\lambda \mu}\right) \mathrm{d} S \\
& + \text { higher order terms. }
\end{aligned}
$$

We now consider the matrix, in which the displacement is a sum of the prebuckled displacement $\boldsymbol{u}^{(0)}$ and a virtual displacement $\boldsymbol{u}$ arising due to the virtual displacement $\boldsymbol{v}$ of the shell. The potential energy density in the matrix is given by

$$
V=\frac{1}{2} A^{i j k l} e_{i j} e_{k l}
$$

where $A^{i j k l}$ is the elasticity tensor and $e_{i j}$ is the strain tensor, given by $e_{i j}=\frac{1}{2}\left(\left.u_{i}\right|_{j}+\left.u_{j}\right|_{i}\right)$. From (3.3), we can see that the change in potential energy density is

$$
\Delta V=\frac{1}{2} A^{i j k l}\left(e_{i j}^{(0)} e_{k l}+e_{i j} e_{k l}^{(0)}+e_{i j} e_{k l}\right),
$$

where $e_{i j}^{(0)}, e_{i j}$ are the strain tensors formed from $\boldsymbol{u}^{(0)}, \boldsymbol{u}$ respectively. The linear and quadratic terms in the change of potential energy of the matrix are thus

$$
\Delta W_{\mathrm{m}}=\iiint_{R>R_{1}} \frac{1}{2} A^{i j k l}\left(e_{i j}^{(0)} e_{k l}+e_{i j} e_{k l}^{(0)}\right) \mathrm{d} V+\iiint_{R>R_{1}} \frac{1}{2} A^{i j k l} e_{i j} e_{k l} \mathrm{~d} V .
$$

Finally, we note that if a hydrostatic pressure $q_{\text {in }}$ is applied to the inner surface of the shell, the potential energy of this loading will be given by

$$
\Delta W_{\mathrm{in}}=-\iint_{R=R_{0}} q_{\mathrm{in}} w \mathrm{~d} S,
$$

which is linear in the virtual displacement.

The total change in potential energy is given by the sum of (3.2), (3.4) and (3.5). We write this as the sum of terms which are linear and quadratic in the virtual displacement, denoted $\Delta W_{1}$ and $\Delta W_{2}$ respectively. Thus

$$
\begin{aligned}
\Delta W_{1}=\iint_{\substack{\text { mid-shell } \\
\text { surface }}}\left({ }^{(0)} n^{\alpha \beta} \theta_{\alpha \beta}+{ }^{(0)} m^{\alpha \beta} \rho_{\alpha \beta}\right) \mathrm{d} S \\
\qquad \\
\qquad \iiint_{R>R_{1}} \frac{1}{2} A^{i j k l}\left(e_{i j}^{(0)} e_{k l}+e_{i j} e_{k l}^{(0)}\right) \mathrm{d} V-\iint_{R=R_{0}} q_{\text {in }} w \mathrm{~d} S,
\end{aligned}
$$


and

$$
\Delta W_{2}=\mathscr{I}_{1}+\mathscr{I}_{2}+\mathscr{I}_{3},
$$

where

$$
\begin{aligned}
& \mathscr{I}_{1}=\iint_{\substack{\text { mid-shell } \\
\text { surface }}} \frac{1}{2}{ }^{(0)} n^{\alpha \beta} w_{, \alpha} w_{, \beta} \mathrm{d} S, \\
& \mathscr{I}_{2}=\iint_{\substack{\text { mid-shell } \\
\text { surface }}}\left(\frac{h}{2} E^{\alpha \beta \lambda \mu} \theta_{\alpha \beta} \theta_{\lambda \mu}+\frac{h^{3}}{24} E^{\alpha \beta \lambda \mu} \rho_{\alpha \beta} \rho_{\lambda \mu}\right) \mathrm{d} S, \\
& \mathscr{I}_{3}=\iiint_{R>R_{1}} \frac{1}{2} A^{i j k l} e_{i j} e_{k l} \mathrm{~d} V .
\end{aligned}
$$

It will be convenient to consider these three contributions to the energy separately. The critical buckling stress is found at a stationary point of $\Delta W_{2}$.

\section{Pre-buckled state of stress}

In this section we will obtain the state of stress in the shell before buckling, which we denoted by (0). As stated in the previous section, this can be found by taking the functional (3.6) and solving the variational problem $\Delta W_{1}=0$ for each possible variation $\boldsymbol{v}$. Ideally we would like a closed-form solution to this problem. However, $\Delta W_{1}$ is a combination of shell strain measures and three-dimensional strains in the matrix, rendering this a difficult task.

It turns out to be easier to solve for the pre-buckled state using linear elasticity and a finite thickness shell, following the method of Love (11). Having found this solution we can then take the limit $h / \widehat{R} \rightarrow 0$ and extract the stress resultant ${ }^{(0)} n^{\alpha \beta}$, which is the only appearance of the pre-buckling solution in the integral $\Delta W_{2}$.

\subsection{Love's method}

Love's method was used by Goodier (14) to solve the problem of a spherical elastic inclusion embedded in a dissimilar elastic matrix. This work was repeated by Liu and Nauman (15) and Bilgen and Insana (16). Mazzullo (17) has built on previous work in the case of a multi-layered inclusion, which is solved numerically due to the large system of equations that results from the analysis. In particular that work looked at the solution of a thick spherical shell surrounding a dissimilar material, all embedded in a matrix of a third material, undergoing uniaxial stress at infinity. On setting the stiffness of the innermost material to zero, we recover the solution to the uniaxial problem outlined in Section 2.

The theory behind Love's method is reviewed in a recent paper by Rahman and Michelitsch (18). It involves constructing displacement fields as a sum of elementary solutions, which take one of three forms - namely the $\phi, \omega$, and $\chi$ solutions. Let $\phi_{n}$, $\omega_{n}$, and $\chi_{n}$ be solid spherical harmonics of order $n$. Then the displacement fields arising from each solution are given by

$$
\begin{aligned}
\boldsymbol{u} & =\nabla \phi_{n}, \\
\boldsymbol{u} & =R^{2} \nabla \omega_{n}+\alpha_{n} R \omega_{n} \boldsymbol{e}_{R}, \\
\boldsymbol{u} & =\nabla \wedge\left(R \chi_{n} \boldsymbol{e}_{R}\right),
\end{aligned}
$$


respectively, where $\boldsymbol{e}_{R}$ is the radial unit vector and

$$
\alpha_{n}=\frac{-2(3 n+1-2(2 n+1) \nu)}{n+5-4 \nu} .
$$

The method involves writing the displacement in each material as a linear combination of these elementary solutions. By considering the displacement and traction boundary conditions a linear system for the coefficients is found, which can be solved (in principle) analytically.

\subsection{Application to the embedded spherical shell}

As stated in Section 2, the state of stress in the pre-buckled shell will be found by superimposing the results of an applied uniaxial compression at infinity and an applied radial compression at infinity. We will not write down the derivation of the solution to these problems; the interested reader may refer to $(\mathbf{1})$.

Applying Love's method to the uniaxial problem with $\left.\tau_{z z}\right|_{\infty}=-q_{z}$, we prescribe the deformation in the matrix to be a superposition of the states arising from the following elementary solutions:

$$
\phi_{-1}=\frac{c_{1}}{R} P_{0}(\mu), \quad \phi_{-3}=\frac{c_{2}}{R^{3}} P_{2}(\mu), \quad \omega_{-3}=\frac{c_{3}}{R^{3}} P_{2}(\mu)
$$

(where $c_{i}$ are undetermined constants, $\mu=\cos \theta$ and $P_{n}(\mu)$ are Legendre polynomials), together with a homogeneous field $\boldsymbol{u}^{\infty}$ which is the displacement given by a constant stress field with only one component, $\tau_{z z}=-q_{z}$. In the shell, we suppose that the deformation is given by the superposition of the states arising from the following elementary solutions:

$$
\begin{aligned}
& \phi_{-1}=\frac{c_{4}}{R} P_{0}(\mu), \quad \phi_{-3}=\frac{c_{5}}{R^{3}} P_{2}(\mu), \quad \omega_{-3}=\frac{c_{6}}{R^{3}} P_{2}(\mu), \\
& \phi_{2}=c_{7} R^{2} P_{2}(\mu), \quad \omega_{2}=c_{8} R^{2} P_{2}(\mu), \quad \omega_{0}=c_{9} P_{0}(\mu),
\end{aligned}
$$

where again $c_{i}$ are undetermined constants. One can thus obtain the displacement and stress in both materials, and solve for the constants by matching displacements and tractions at $R=R_{1}$, and setting the traction at $R=R_{0}$ to zero. The resulting expressions for the constants $c_{i}$ are somewhat unwieldy so we do not record them here. However, for future reference we note that the component $\tau_{\theta \theta}$ of stress in the shell is given by

$$
\begin{aligned}
\tau_{\theta \theta}=2 G_{\mathrm{s}}\left(-\frac{c_{4}}{R^{3}}+\alpha_{0}^{(\mathrm{s})} c_{9}+\frac{c_{5}}{R^{5}}+\frac{c_{6}}{R^{3}}+c_{7}+c_{8} R^{2}+\frac{3 \nu_{\mathrm{s}} \alpha_{0}^{(\mathrm{s})} c_{9}}{1-2 \nu_{\mathrm{s}}}\right) P_{0}(\mu) \\
+2 G_{\mathrm{s}}\left[-\frac{7 c_{5}}{R^{5}-2 c_{7}}+\frac{\left(-7+\alpha_{-3}^{(\mathrm{s})}\right) c_{6}}{R^{3}}+\left(-2+\alpha_{2}^{(\mathrm{s})}\right) R^{2} c_{8}\right. \\
\left.+\frac{\nu_{\mathrm{s}}}{1-2 \nu_{\mathrm{s}}}\left(-\frac{6 c_{6}}{R^{3}}+\left(4+5 \alpha_{2}^{(\mathrm{s})}\right) c_{8} R^{2}\right)\right] P_{2}(\mu),
\end{aligned}
$$

where $\alpha_{n}^{(\mathrm{s})}$ is given by substituting the Poisson ratio of the shell, $\nu_{\mathrm{s}}$, into (4.1). 
For the radial compression at infinity, $\left.\tau_{R R}\right|_{\infty}=-q_{R}$ and $\left.\tau_{R R}\right|_{R_{0}}=-q_{\text {in }}$. The prebuckling displacement will be purely radial, with

$$
\boldsymbol{u}=\left(A_{\mathrm{s}} R+\frac{B_{\mathrm{s}}}{R^{2}}\right) \boldsymbol{e}_{R}, \quad \boldsymbol{u}=\left(A_{\mathrm{m}} R+\frac{B_{\mathrm{m}}}{R^{2}}\right) \boldsymbol{e}_{R}
$$

in the shell and the matrix respectively (11). Again we can find the stresses in both materials, and match displacements and tractions at the interface. We merely note here that

$$
\tau_{\theta \theta}=\frac{2 G_{\mathrm{s}}\left(1+\nu_{\mathrm{s}}\right)}{1-2 \nu_{\mathrm{s}}} A_{\mathrm{s}}\left(1+\frac{R_{0}^{3}}{2 R^{3}}\right)+\frac{q_{\mathrm{in}} R_{0}^{3}}{2 R^{3}}
$$

where

$$
A_{\mathrm{s}}=\frac{\frac{q_{\mathrm{in}} R_{0}^{3}}{4 R_{1}^{3}}\left(\frac{1}{G_{\mathrm{m}}}-\frac{1}{G_{\mathrm{s}}}\right)-\frac{3 q_{R}\left(1-\nu_{\mathrm{m}}\right)}{4 G_{\mathrm{m}}\left(1+\nu_{\mathrm{m}}\right)}}{\left\{1+\frac{\left(1+\nu_{\mathrm{s}}\right)}{2\left(1-2 \nu_{\mathrm{s}}\right)}\left[\frac{R_{0}^{3}}{R_{1}^{3}}+\frac{G_{\mathrm{s}}}{G_{\mathrm{m}}}\left(1-\frac{R_{0}^{3}}{R_{1}^{3}}\right)\right]\right\}} .
$$

\subsection{Finding the stress resultant}

We now use the solution to the pre-buckled state in the shell to find the stress resultant ${ }^{(0)} n^{\alpha \beta}$ required in (3.8). In fact, for axisymmetric buckling patterns, we have $w_{, 2}=$ $\partial w / \partial \phi=0$, so the only stress resultant we need is ${ }^{(0)} n^{11}$. This term is related to the stress component $\tau_{\theta \theta}$ in the shell, which is given by the sum of (4.2) and (4.3).

From the results in Niordson (13) applied to a spherical shell,

$$
\left.n^{11} \sim \int_{-h / 2}^{h / 2} \frac{1}{\widehat{R}^{2}}\left(1+\frac{\rho}{\widehat{R}}\right) \tau_{\theta \theta} \mathrm{d} \rho \sim \frac{h}{\widehat{R}^{2}} \lim _{h / \widehat{R} \rightarrow 0} \tau_{\theta \theta}\right|_{R=\widehat{R}} \quad \text { as } \quad \frac{h}{\widehat{R}} \rightarrow 0,
$$

where $R=\widehat{R}+\rho$. To determine $\lim _{h / \widehat{R} \rightarrow 0} \tau_{\theta \theta}$ we evaluate the sum of (4.2) and (4.3) at $R=\widehat{R}$, with the constants $c_{i}$ and $A_{\mathrm{s}}$ replaced by their values in the limit $h / \widehat{R} \rightarrow 0$. Having found these quantities, we obtain

$$
{ }^{(0)} n^{11}=p_{0} P_{0}(\mu)+p_{2} P_{2}(\mu),
$$

where

$$
\begin{aligned}
p_{0}= & \frac{q_{z} h G_{\mathrm{s}}}{2 \widehat{R}^{2} G_{\mathrm{m}}} \frac{\left(1-\nu_{\mathrm{m}}\right)\left(-5 \nu_{\mathrm{m}}+15 \nu_{\mathrm{m}} \nu_{\mathrm{s}}-17+3 \nu_{\mathrm{s}}\right)}{\left(1-\nu_{\mathrm{s}}\right)\left(7-5 \nu_{\mathrm{m}}\right)\left(1+\nu_{\mathrm{m}}\right)} \\
& \quad+\frac{h\left(1+\nu_{\mathrm{s}}\right)}{2 \widehat{R}^{2}\left(1-\nu_{\mathrm{s}}\right)}\left[q_{\mathrm{in}}\left(\frac{G_{\mathrm{s}}}{G_{\mathrm{m}}}-1\right)-3 q_{R} \frac{G_{\mathrm{s}}}{G_{\mathrm{m}}} \frac{\left(1-\nu_{\mathrm{m}}\right)}{\left(1+\nu_{\mathrm{m}}\right)}\right]+\frac{q_{\mathrm{in}} h}{2 \widehat{R}^{2}} \\
p_{2}= & \frac{10 q_{z} h G_{\mathrm{s}}\left(1-\nu_{\mathrm{m}}\right)}{\widehat{R}^{2} G_{\mathrm{m}}\left(1-\nu_{\mathrm{s}}\right)\left(7-5 \nu_{\mathrm{m}}\right)}
\end{aligned}
$$

\subsection{Interpretation}

Given the stress resultant calculated in the previous section, it is instructive to find which regions of the shell are in compression and tension. We have

$$
{ }^{(\mathrm{I})} n^{11}=p_{0}+\frac{p_{2}}{4}+\frac{3 p_{2}}{4} \cos 2 \theta
$$




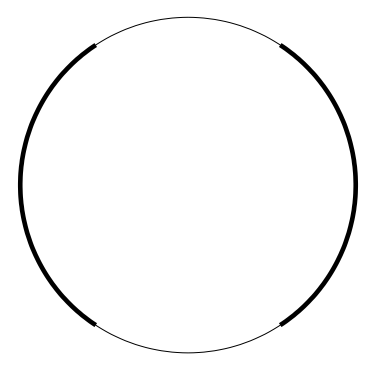

Fig. 2 Areas of the spherical shell in compression (thick) and tension (thin), for a uniaxial compression in the $z$-direction at infinity, with $\nu_{\mathrm{s}}=0.35$ and $\nu_{\mathrm{m}}=0.45$.

At the transition point between tension and compression, we have $n^{11}=0$, giving

$$
\theta=\left\{\frac{1}{2} \cos ^{-1}\left[-\frac{4}{3 p_{2}}\left(p_{0}+\frac{p_{2}}{4}\right)\right], \pi-\frac{1}{2} \cos ^{-1}\left[-\frac{4}{3 p_{2}}\left(p_{0}+\frac{p_{2}}{4}\right)\right]\right\}
$$

assuming that

$$
\left|\frac{4}{3 p_{2}}\left(p_{0}+\frac{p_{2}}{4}\right)\right|<1
$$

In the case that $q_{z}>0$ (compression at infinity), we have that ${ }^{(\mathrm{I})} n^{11}<0$ in between the values in (4.5), assuming condition (4.6) still holds. (The converse is true if $q_{z}<0$.)

Note in particular that if $q_{R}=q_{\text {in }}=0$, the values in (4.5) depend only on the Poisson ratios $\nu_{\mathrm{m}}$ and $\nu_{\mathrm{s}}$. For example, taking the values $\nu_{\mathrm{s}}=0.35, \nu_{\mathrm{m}}=0.45$ and setting $q_{R}=$ $q_{\text {in }}=0$, we find that we are in compression for $\theta \in(0.582,2.559)$, independently of the shear moduli of the materials and the magnitude of the applied stress at infinity. The region of compression is shown as the thick curve in Figure 2 (the thin curve representing areas in tension).

\section{Stationarity of the quadratic variation $\Delta W_{2}$}

\subsection{The Rayleigh-Ritz method}

We now consider the problem of finding the critical load through the stationarity point of (3.7). Since we are limiting our analysis to axisymmetric buckling patterns, we can write $\boldsymbol{v}=v_{R} \boldsymbol{e}_{R}+v_{\theta} \boldsymbol{e}_{\theta}$, where $v_{R}, v_{\theta}$ are functions of $R$ and $\theta$ only. Note that these are the physical components of the shell displacement, which can be related to the components referred to base vectors by $v_{1}=\widehat{R} v_{\theta}$ and $w=v_{R}$.

To find the stationary value of (3.7), we will use the Rayleigh-Ritz approach (19) which involves writing the virtual displacement as an infinite series,

$$
v_{R}=\sum_{n=0}^{\infty} \mathscr{U}_{n} P_{n}(\mu) \quad v_{\theta}=\sum_{n=1}^{\infty} \mathscr{V}_{n} P_{n}^{1}(\mu)
$$

or equivalently

$$
w=\sum_{n=0}^{\infty} \mathscr{U}_{n} P_{n}(\mu), \quad v_{1}=\sum_{n=1}^{\infty} \widehat{R} \mathscr{V}_{n} P_{n}^{1}(\mu),
$$


where $P_{n}^{1}(\mu)$ is an associated Legendre function; these are defined by the formula

$$
P_{n}^{m}(\mu)=(-1)^{m}\left(1-\mu^{2}\right)^{m / 2} \frac{\mathrm{d}^{m} P_{n}}{\mathrm{~d} \mu^{m}} .
$$

The coefficients are then found by solving

$$
\frac{\partial}{\partial \mathscr{U}_{n}} \Delta W_{2}=0, \quad \frac{\partial}{\partial \mathscr{W}_{n}} \Delta W_{2}=0
$$

We will get an infinite system of linear equations whose determinant must be set to zero for a nonzero buckling deformation. The critical value for the applied stress at infinity will be found from this condition.

The identities involving Legendre polynomials and associated Legendre functions that are necessary for this analysis can be found, for example, in Lebedev's treatise (20). Chief among these identities is the orthogonality condition,

$$
\int_{-1}^{1} P_{k}^{m}(\mu) P_{l}^{m}(\mu) \mathrm{d} \mu=\frac{2}{2 k+1} \frac{(k+m) !}{(k-m) !} \delta_{k l} .
$$

5.2 Application of the Rayleigh-Ritz method to $\Delta W_{2}$

Consider first $\mathscr{I}_{1}$. Given that $w$ is independent of the coordinate $\phi$, from (3.8) we have that

$$
\mathscr{I}_{1}=\iint_{\text {shell }} \frac{{ }^{(0)} n^{11}}{2}\left(\frac{\mathrm{d} w}{\mathrm{~d} \theta}\right)^{2} \mathrm{~d} S
$$

'shell' means the mid-shell surface. The stress resultant is given in general by (4.4), but for simplicity we will consider two cases only: firstly $q_{z}=q_{\infty}, q_{R}=0$ and $q_{\text {in }}=0$; and secondly $q_{z}=0, q_{R}=q_{\infty}$ and $q_{\text {in }}=0$. Thus ${ }^{(0)} n^{11}=q_{\infty}\left(p_{0} P_{0}(\mu)+p_{2} P_{2}(\mu)\right)$, where for the first case we redefine

$$
\begin{aligned}
& p_{0}=\frac{h G_{\mathrm{s}}}{2 \widehat{R}^{2} G_{\mathrm{m}}} \frac{\left(1-\nu_{\mathrm{m}}\right)\left(-5 \nu_{\mathrm{m}}+15 \nu_{\mathrm{m}} \nu_{\mathrm{s}}-17+3 \nu_{\mathrm{s}}\right)}{\left(1-\nu_{\mathrm{s}}\right)\left(7-5 \nu_{\mathrm{m}}\right)\left(1+\nu_{\mathrm{m}}\right)}, \\
& p_{2}=\frac{10 h G_{\mathrm{s}}\left(1-\nu_{\mathrm{m}}\right)}{\widehat{R}^{2} G_{\mathrm{m}}\left(1-\nu_{\mathrm{s}}\right)\left(7-5 \nu_{\mathrm{m}}\right)}
\end{aligned}
$$

and for the second case

$$
p_{0}=-\frac{3 h G_{\mathrm{s}}\left(1+\nu_{\mathrm{s}}\right)\left(1-\nu_{\mathrm{m}}\right)}{2 \widehat{R}^{2} G_{\mathrm{m}}\left(1+\nu_{\mathrm{m}}\right)\left(1-\nu_{\mathrm{s}}\right)}, \quad p_{2}=0
$$

Then

$$
\mathscr{I}_{1}=\iint_{\text {shell }} \frac{q_{\infty}}{2}\left(p_{0}+p_{2} P_{2}(\mu)\right)\left(\frac{\mathrm{d} w}{\mathrm{~d} \theta}\right)^{2} \mathrm{~d} S .
$$

Now consider $\mathscr{I}_{2}$ from (3.9). Koiter (10) employed the van der Neut substitution,

$$
v_{\alpha}=\psi_{, \alpha}+\varepsilon_{\alpha \lambda} a^{\lambda \mu} \chi_{, \mu},
$$


where $\varepsilon_{\alpha \lambda}$ is the surface alternating tensor and $\psi, \chi$ are functions to be determined. Under our assumption of axisymmetry, this simplifies to $v_{\alpha}=\psi_{, \alpha}$, where from $(5.2)_{2}$ we have

$$
\psi=\sum_{n=0}^{\infty}\left(\widehat{R} \mathscr{V}_{n}\right) P_{n}(\mu),
$$

with $\mathscr{V}_{0}$ arbitrary (we will set it to zero without loss of generality). This substitution eventually allows us, following Koiter, to write

$$
\mathscr{I}_{2}=\frac{h G_{\mathrm{s}}}{1-\nu_{\mathrm{s}}} \iint_{\text {shell }}\left[\left(\nabla^{2} \psi-\left(1+\nu_{\mathrm{s}}\right) \frac{w}{\widehat{R}}\right)^{2}+\left(1-\nu_{\mathrm{s}}^{2}\right) \frac{w^{2}}{\widehat{R}^{2}}+\frac{h^{2}}{12}\left(\nabla^{2} w\right)^{2}\right] \mathrm{d} S .
$$

Finally, consider the integral $\mathscr{I}_{3}$, from (3.10). The stress tensor is given by $\tau^{i j}=A^{i j k l} e_{k l}$, so that

$$
\mathscr{I}_{3}=\left.\frac{1}{2} \iiint_{R>R_{1}} \tau^{i j} u_{i}\right|_{j} \mathrm{~d} V
$$

on using the symmetry of the stress tensor and the definition of the strain tensor. Then, by the divergence theorem and the equilibrium equation for the stress tensor, we have

$$
\mathscr{I}_{3}=-\frac{1}{2} \iint_{\partial V}\left(\tau^{11} u_{1}+\tau^{12} u_{2}\right) \mathrm{d} S
$$

where $\partial V$ is the internal boundary of the region, $R=R_{1}$, and we have used the fact that the only nonzero component of the normal is $n_{1}=1$. The displacement components $u_{1}$ and $u_{2}$ here correspond to the displacement of the outer surface of the shell, which can be replaced by the middle-surface displacement components to leading order. Thus, writing the stresses in physical components, we obtain

$$
\mathscr{I}_{3}=-\frac{1}{2} \iint_{R=R_{1}}\left(\tau_{R R} w+\tau_{R \theta} v_{\theta}\right) \mathrm{d} S .
$$

Combining the integrals $\mathscr{I}_{1}, \mathscr{I}_{2}$ and $\mathscr{I}_{3}$, we have

$$
\begin{aligned}
\Delta W_{2}=\iint_{\text {shell }}\left\{\frac{q_{\infty}}{2}\left(p_{0}+p_{2} P_{2}(\mu)\right)\left(\frac{\mathrm{d} w}{\mathrm{~d} \theta}\right)^{2}+\frac{h G_{\mathrm{s}}}{1-\nu_{\mathrm{s}}}\left[\left(\nabla^{2} \psi-\left(1+\nu_{\mathrm{s}}\right) \frac{w}{\widehat{R}}\right)^{2}\right.\right. \\
\left.\left.+\left(1-\nu_{\mathrm{s}}^{2}\right) \frac{w^{2}}{\widehat{R}^{2}}+\frac{h^{2}}{12}\left(\nabla^{2} w\right)^{2}\right]\right\} \mathrm{d} S-\frac{1}{2} \iint_{R=R_{1}}\left(\tau_{R R} w+\tau_{R \theta} v_{\theta}\right) \mathrm{d} S .
\end{aligned}
$$

5.3 The functional in terms of the Legendre coefficients

Substituting $(5.2)_{1}$ into $\mathscr{I}_{1}$, recalling that $P_{2}(\mu)=\left(3 \mu^{2}-1\right) / 2$, and simplifying using (5.4) and other standard identities (the reader may refer to (1) for the details) we find

$$
\begin{aligned}
\mathscr{I}_{1}=\pi q_{\infty} \widehat{R}^{2} \sum_{n=1}^{\infty}[ & \left\{3 p_{2}\left(\frac{n^{2}(n+1)(n+2)}{(2 n+1)^{2}(2 n+3)}+\frac{(n-1) n(n+1)^{2}}{(2 n-1)(2 n+1)^{2}}\right)\right. \\
& \left.\left.+\left(2 p_{0}-p_{2}\right) \frac{n(n+1)}{2 n+1}\right\} \mathscr{U}_{n}^{2}+\frac{6 p_{2} n(n+1)(n+2)(n+3)}{(2 n+1)(2 n+3)(2 n+5)} \mathscr{U}_{n} \mathscr{U}_{n+2}\right] .
\end{aligned}
$$


Since

we have

$$
\nabla^{2} \psi=\frac{1}{\widehat{R}^{2}}(\psi, 11+\cot \theta \psi, 1), \quad \nabla^{2} w=\frac{1}{\widehat{R}^{2}}\left(w_{, 11}+\cot \theta w_{, 1}\right),
$$

$$
\nabla^{2} \psi=-\sum_{n=0}^{\infty} \frac{n(n+1) \mathscr{V}_{n}}{\widehat{R}} P_{n}(\mu), \quad \nabla^{2} w=-\sum_{n=0}^{\infty} \frac{n(n+1) \mathscr{U}_{n}}{\widehat{R}^{2}} P_{n}(\mu),
$$

which gives, after using (5.4),

$$
\begin{aligned}
\mathscr{I}_{2}=\frac{4 \pi h G_{\mathrm{s}}}{\widehat{R}^{2}\left(1-\nu_{\mathrm{s}}\right)} \sum_{n=0}^{\infty} \frac{1}{2 n+1}\left[\left(n(n+1) \mathscr{V}_{n}+\left(1+\nu_{\mathrm{s}}\right) \mathscr{U}_{n}\right)^{2} \widehat{R}^{2}\right. & \\
& \left.+\left(1-\nu_{\mathrm{s}}^{2}\right) \widehat{R}^{2} \mathscr{U}_{n}^{2}+\frac{h^{2} n^{2}(n+1)^{2} \mathscr{U}_{n}^{2}}{12}\right] .
\end{aligned}
$$

In order to calculate $\mathscr{I}_{3}$, we need to determine the displacement field $\boldsymbol{u}$ that is induced in the elastic matrix from the virtual deformation $\boldsymbol{v}$ of the shell. This is found by solving the elasticity equations for $\boldsymbol{u}$ with a displacement boundary condition $\left.\boldsymbol{u}\right|_{R=R_{1}}=\boldsymbol{v}$ (since the displacement on the outer shell surface is approximately equal to the mid-surface displacement $\boldsymbol{v}$ ) and zero stress at infinity. This problem has been solved by Lur'e (21). Firstly the boundary displacement is decomposed into a series of homogeneous surface vector spherical harmonics,

$$
\boldsymbol{v}=\sum_{n=0}^{\infty} \boldsymbol{Y}_{n}(\theta, \phi)
$$

Then the vector

$$
\boldsymbol{U}_{-n-1}=\left(\frac{R_{1}}{R}\right)^{n+1} \boldsymbol{Y}_{n}(\theta, \phi)
$$

is formed, which gives us the final solution

$$
\boldsymbol{u}=\sum_{n=0}^{\infty}\left[\boldsymbol{U}_{-n-1}-\frac{1}{2}\left(R_{1}^{2}-R^{2}\right) \frac{\nabla\left(\nabla \cdot \boldsymbol{U}_{-n-1}\right)}{\left(3-4 \nu_{\mathrm{m}}\right)(n+1)+2\left(1-\nu_{\mathrm{m}}\right)}\right]
$$

The problem we now have is to translate between the series (5.9) in spherical harmonics and the series (5.1) in Legendre functions. Now

$$
\begin{aligned}
\boldsymbol{Y}_{n} & =\alpha_{n} P_{n}^{1}(\mu)\left(\cos \phi \boldsymbol{e}_{x}+\sin \phi \boldsymbol{e}_{y}\right)+\beta_{n} P_{n}(\mu) \boldsymbol{e}_{z} \\
& =\left(\alpha_{n} \sqrt{1-\mu^{2}} P_{n}^{1}(\mu)+\beta_{n} \mu P_{n}(\mu)\right) \boldsymbol{e}_{R}+\left(\alpha_{n} \mu P_{n}^{1}(\mu)-\beta_{n} \sqrt{1-\mu^{2}} P_{n}(\mu)\right) \boldsymbol{e}_{\theta} .
\end{aligned}
$$

Using standard identities we find, after some manipulation,

$\boldsymbol{Y}_{n}=\left(n\left(\gamma_{n}-\alpha_{n}\right) P_{n-1}(\mu)+(n+1) \gamma_{n} P_{n+1}(\mu)\right) \boldsymbol{e}_{R}+\left(\left(\alpha_{n}-\gamma_{n}\right) P_{n-1}^{1}(\mu)+\gamma_{n} P_{n+1}^{1}(\mu)\right) \boldsymbol{e}_{\theta}$,

where $\gamma_{n}=\left(n \alpha_{n}+\beta_{n}\right) /(2 n+1)$. Thus

$$
\boldsymbol{v}=\sum_{n=0}^{\infty}\left[n\left(\gamma_{n}-\alpha_{n}\right) P_{n-1}+(n+1) \gamma_{n} P_{n+1}\right] \boldsymbol{e}_{R}+\sum_{n=0}^{\infty}\left[\left(\alpha_{n}-\gamma_{n}\right) P_{n-1}^{1}+\gamma_{n} P_{n+1}^{1}\right] \boldsymbol{e}_{\theta}
$$




$$
\begin{gathered}
=\sum_{m=0}^{\infty}\left[(m+1)\left(\gamma_{m+1}-\alpha_{m+1}\right)+m \gamma_{m-1}\right] P_{m}(\mu) \boldsymbol{e}_{R} \\
+\sum_{m=1}^{\infty}\left[\alpha_{m+1}-\gamma_{m+1}+\gamma_{m-1}\right] P_{m}^{1}(\mu) \boldsymbol{e}_{\theta}
\end{gathered}
$$

Comparing with (5.1) we obtain

$$
\mathscr{U}_{n}=(n+1)\left(\gamma_{n+1}-\alpha_{n+1}\right)+n \gamma_{n-1}, \quad \mathscr{V}_{n}=\alpha_{n+1}-\gamma_{n+1}+\gamma_{n-1},
$$

or

$$
\gamma_{n}-\alpha_{n}=\frac{\mathscr{U}_{n-1}-(n-1) \mathscr{V}_{n-1}}{2 n-1}, \quad \gamma_{n}=\frac{\mathscr{U}_{n+1}+(n+2) \mathscr{V}_{n+1}}{2 n+3}
$$

Now, using (5.12), (5.10), (5.11) and various identities involving Legendre functions we find, after some manipulation,

$$
\begin{aligned}
\boldsymbol{u}=\sum_{m=0}^{\infty}\left[A_{m}\left(\frac{R_{1}}{R}\right)^{m+2}+B_{m}\right. & \left.\left(\frac{R_{1}}{R}\right)^{m}\right] P_{m}(\mu) \boldsymbol{e}_{R} \\
& +\sum_{m=1}^{\infty}\left[C_{m}\left(\frac{R_{1}}{R}\right)^{m+2}+D_{m}\left(\frac{R_{1}}{R}\right)^{m}\right] P_{m}^{1}(\mu) \boldsymbol{e}_{\theta},
\end{aligned}
$$

where $A_{n}+B_{n}=\mathscr{U}_{n}, C_{n}+D_{n}=\mathscr{V}_{n}, A_{n}=-(n+1) C_{n}$, and

$$
C_{n}=\frac{1}{2 n+1}\left\{n \mathscr{V}_{n}-\mathscr{U}_{n}+\frac{n(2 n-1)}{2}\left[\frac{\mathscr{U}_{n}+(n+1) \mathscr{V}_{n}}{\left(3-4 \nu_{\mathrm{m}}\right) n+2\left(1-\nu_{\mathrm{m}}\right)}\right]\right\} .
$$

Now we use the stress-strain relations in spherical polar coordinates to obtain the stress components from the displacement (5.13). On substituting these into $\mathscr{I}_{3}$, we finally deduce that

$$
\begin{aligned}
\mathscr{I}_{3}=4 \pi G_{\mathrm{m}} R_{1} \sum_{n=0}^{\infty}\left\{\frac{\mathscr{U}_{n}}{2 n+1}\right. & {\left[\frac{\nu_{\mathrm{m}}}{1-2 \nu_{\mathrm{m}}}\left((n-2) \mathscr{U}_{n}-2(n+1) C_{n}+n(n+1) \mathscr{V}_{n}\right)\right.} \\
& \left.\left.\quad+n \mathscr{U}_{n}-2(n+1) C_{n}\right]+\frac{n(n+1) \mathscr{V}_{n}}{2(2 n+1)}\left[(n+1) \mathscr{V}_{n}+2 C_{n}-\mathscr{U}_{n}\right]\right\},
\end{aligned}
$$

where $C_{n}$ is given by $(5.14)$.

\section{The eigenvalue problem}

Having found $\mathscr{I}_{1}, \mathscr{I}_{2}$ and $\mathscr{I}_{3}$ in terms of $\mathscr{U}_{n}$ and $\mathscr{V}_{n}$ we can now apply the buckling criteria (5.3). Firstly, we note that $(5.3)_{2}$ gives

$$
\mathscr{V}_{n}=\mu_{n} \mathscr{U}_{n}
$$

where

$$
\begin{array}{r}
\mu_{n}=-\left\{\frac{2 h n(n+1) G_{\mathrm{s}}}{R_{1}\left(1-\nu_{\mathrm{s}}\right) G_{\mathrm{m}}}+(n+1)+2 E_{n}\right\}^{-1}\left\{\frac{2 h\left(1+\nu_{\mathrm{s}}\right) G_{\mathrm{s}}}{R_{1}\left(1-\nu_{\mathrm{s}}\right) G_{\mathrm{m}}}-\frac{2 E_{n}\left(1-\nu_{\mathrm{m}}\right)}{n\left(1-2 \nu_{\mathrm{m}}\right)}\right. \\
\left.+\frac{1}{2 n+1}\left(\frac{n(2 n-1)}{2\left(\left(3-4 \nu_{\mathrm{m}}\right) n+2\left(1-\nu_{\mathrm{m}}\right)\right)}-1\right)-\frac{1}{2}+\frac{\nu_{\mathrm{m}}}{1-2 \nu_{\mathrm{m}}}\right\}
\end{array}
$$


with $\mu_{0}=0$. In this expression, $E_{n}$ is the quantity

$$
E_{n}=\frac{\partial C_{n}}{\partial \mathscr{V}_{n}}=\frac{1}{2 n+1}\left[n+\frac{n(n+1)(2 n-1)}{2\left(\left(3-4 \nu_{\mathrm{m}}\right) n+2\left(1-\nu_{\mathrm{m}}\right)\right)}\right] .
$$

Substituting (6.1) into the expressions for $\mathscr{I}_{1}$ to $\mathscr{I}_{3}$ gives

$$
\Delta W_{2}=\sum_{n=0}^{\infty}\left[\left(a_{n} q_{\infty}+b_{n}\right) \mathscr{U}_{n}^{2}+c_{n} q_{\infty} \mathscr{U}_{n} \mathscr{U}_{n+2}\right] ;
$$

the coefficients are given by

$$
\begin{aligned}
a_{n}= & \pi \widehat{R}^{2}\left\{\left(2 p_{0}-p_{2}\right) \frac{n(n+1)}{2 n+1}+3 p_{2}\left[\frac{n^{2}(n+1)(n+2)}{(2 n+1)^{2}(2 n+3)}+\frac{(n-1) n(n+1)^{2}}{(2 n-1)(2 n+1)^{2}}\right]\right\} \\
b_{n}= & \frac{4 \pi h G_{\mathrm{s}}}{\widehat{R}^{2}\left(1-\nu_{\mathrm{s}}\right)(2 n+1)}\left[\left(n(n+1) \mu_{n}+1+\nu_{\mathrm{s}}\right)^{2} \widehat{R}^{2}+\left(1-\nu_{\mathrm{s}}^{2}\right) \widehat{R}^{2}+h^{2} n^{2}(n+1)^{2} / 12\right] \\
& +\frac{4 \pi G_{\mathrm{m}} R_{1}}{2 n+1}\left[n-2(n+1) F_{n}+\frac{\nu_{\mathrm{m}}}{1-2 \nu_{\mathrm{m}}}\left(n-2-2(n+1) F_{n}+n(n+1) \mu_{n}\right)\right. \\
\left.\quad+\frac{n(n+1) \mu_{n}}{2}\left((n+1) \mu_{n}+2 F_{n}-1\right)\right] & \\
c_{n}= & \frac{6 \pi \widehat{R}^{2} p_{2} n(n+1)(n+2)(n+3)}{(2 n+1)(2 n+3)(2 n+5)}
\end{aligned}
$$

where

$$
F_{n}=\frac{C_{n}}{\mathscr{U}_{n}}=\frac{1}{2 n+1}\left\{n \mu_{n}-1+\frac{n(2 n-1)}{2}\left[\frac{1+(n+1) \mu_{n}}{\left(3-4 \nu_{\mathrm{m}}\right) n+2\left(1-\nu_{\mathrm{m}}\right)}\right]\right\} .
$$

Now $(5.3)_{1}$ gives the system of equations

$$
\left(-\frac{a_{n}}{b_{n}}-\lambda\right) \mathscr{U}_{n}-\frac{c_{n-2}}{2 b_{n}} \mathscr{U}_{n-2}-\frac{c_{n}}{2 b_{n}} \mathscr{U}_{n+2}=0
$$

for $\mathscr{U}_{n}$, where $c_{-2}$ and $c_{-1}$ are both zero, and $\lambda=1 / q_{\infty}$. We see that the even and odd coefficients decouple, so that we may write

$$
\begin{aligned}
& \left(-\frac{a_{2 n-1}}{b_{2 n-1}}-\lambda\right) \mathscr{U}_{n}^{\text {odd }}-\frac{c_{2 n-3}}{2 b_{2 n-1}} \mathscr{U}_{n-1}^{\text {odd }}-\frac{c_{2 n-1}}{2 b_{2 n-1}} \mathscr{U}_{n+1}^{\text {odd }}=0 \\
& \left(-\frac{a_{2 n-2}}{b_{2 n-2}}-\lambda\right) \mathscr{U}_{n}^{\text {even }}-\frac{c_{2 n-4}}{2 b_{2 n-2}} \mathscr{U}_{n-1}^{\text {even }}-\frac{c_{2 n-2}}{2 b_{2 n-2}} \mathscr{U}_{n+1}^{\text {even }}=0
\end{aligned}
$$

for $n=1,2, \ldots$, where $\mathscr{U}_{n}^{\text {odd }}=\mathscr{U}_{2 n-1}$ and $\mathscr{U}_{n}^{\text {even }}=\mathscr{U}_{2 n-2}$. The expressions $(6.5)-(6.6)$ comprise two eigenvalue problems for infinite tridiagonal matrices:

$$
\left(\boldsymbol{A}^{\text {odd }}-\lambda \boldsymbol{I}\right) \mathscr{U}^{\text {odd }}=\mathbf{0}, \quad\left(\boldsymbol{A}^{\text {even }}-\lambda \boldsymbol{I}\right) \mathscr{U}^{\text {even }}=\mathbf{0},
$$

which can be solved numerically. 


\section{Results}

We present results for two modes of deformation, namely uniaxial compression at infinity, where the stress field at infinity has only the component $\tau_{z z}=-q_{\infty}$, and a hydrostatic compression at infinity, for which $\tau_{R R}=-q_{\infty}$. We will consider each case in turn.

\subsection{Uniaxial compression}

The infinite systems of the previous section are truncated and solved numerically using (5.5) and (5.6) for $p_{0}$ and $p_{2}$. Without loss of generality we can set $G_{\mathrm{m}}=1, \widehat{R}=1$ by rescaling. We assume that $G_{\mathrm{s}}=100 G_{\mathrm{m}}$ and $h=0.02 \widehat{R}$, and that the Poisson ratios of the two materials are $\nu_{\mathrm{m}}=0.45, \nu_{\mathrm{s}}=0.35$. The truncation point of the infinite system must be chosen carefully so as not to 'lose' any information from the system; we choose the point such that if the number of terms is doubled the solution remains the same to within a certain tolerance. We will be searching for the lowest positive value of $q_{\infty}$, in order to find the first point at which a buckled solution bifurcates from the equilibrium solution (considering a gradual quasisteady loading of the material). This corresponds to finding the largest eigenvalue $\lambda$. Considering even and odd buckling modes separately, the largest positive eigenvalue in both cases is $\lambda=18.18$, giving a lowest critical compressive stress at infinity of $q_{\infty}=0.0550$. The corresponding eigenvectors in each case give us the constants $\mathscr{U}_{n}$, and hence from (6.1) the constants $\mathscr{V}_{n}$. We substitute these values of the coefficients into (5.1) to determine the displacement components of the characteristic buckling pattern which would occur at the critical buckling stress. These buckling patterns are shown in Figure 3.

(a)

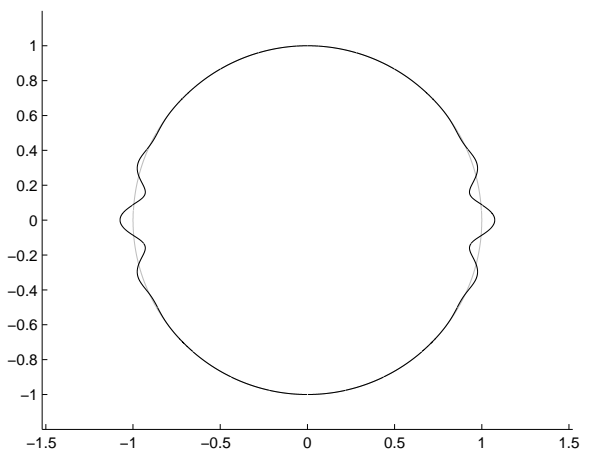

(b)

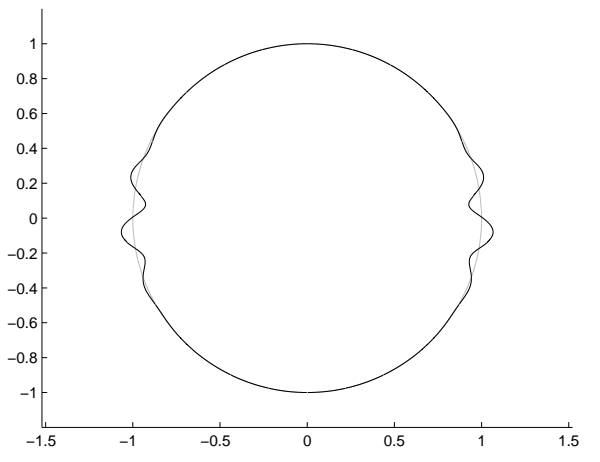

Fig. 3 Buckling patterns for the largest positive eigenvalue: (a) even, (b) odd.

It may seem surprising that the spheres buckle around the equator. After all, by common experience if a spherical shell is placed between two flat plates and compressed, which is a superficially similar mode of deformation, the spheres tend to buckle at the poles. However, compressing spherical shells between flat plates induces a different pattern of stresses in the shell from embedding them in an elastic material. An embedded spherical shell, under uniaxial compression at infinity, is in compression (in the $\tau_{\theta \theta}$ component) around the equator 
while in tension around the poles (see Figure 2); this is why buckling occurs around the equator. Conversely, placing a shell between two flat plates and compressing it results in the region of highest compressive stress — and hence buckling — being around the poles.

One should therefore be wary of modelling the buckling of embedded shells by sandwiching them between flat plates. This approach does have its uses, however: if the shells are not bonded to the elastic matrix, and the matrix is much more compliant than the shell (as is the case here, since $G_{\mathrm{s}}=100 G_{\mathrm{m}}$ ), then the shells will only be in contact with the matrix at the poles, mimicking the sandwiching approach. The likely true configuration of the spheres is partial bonding, which would require us to solve a coupled delamination problem for the shells.

We note that since the critical buckling stress $q_{\infty}$ remains considerably smaller than the matrix shear modulus $G_{\mathrm{m}}$, it can reasonably be assumed that the strains in the matrix remain in the linear regime.

It is interesting to note the behaviour of the solution to the eigenvalue problem as we vary the parameters. In particular, consider the thickness ratio $h / \widehat{R}$. On keeping the other parameters fixed, the effect that changing this ratio has on the critical stress $q_{\infty}$ can be seen in Figure 4(a). We notice that as the ratio tends to zero the critical stress tends to a fixed value. We can also determine what effect this has on the buckling pattern. As shown in Figure 5 , as $h / \widehat{R} \rightarrow 0$ the number of oscillations in the pattern increases while the region over which buckling occurs becomes ever smaller.

The second parameter that we consider is the ratio of shear moduli, $G_{\mathrm{S}} / G_{\mathrm{m}}$. In the limit as this quantity tends to zero, holding the other constants at the values chosen previously, we see from Figure $4(\mathrm{~b})$ that the critical stress $q_{\infty}$ blows up. The effect on the buckling pattern in this limit is similar to that seen in Figure 5.
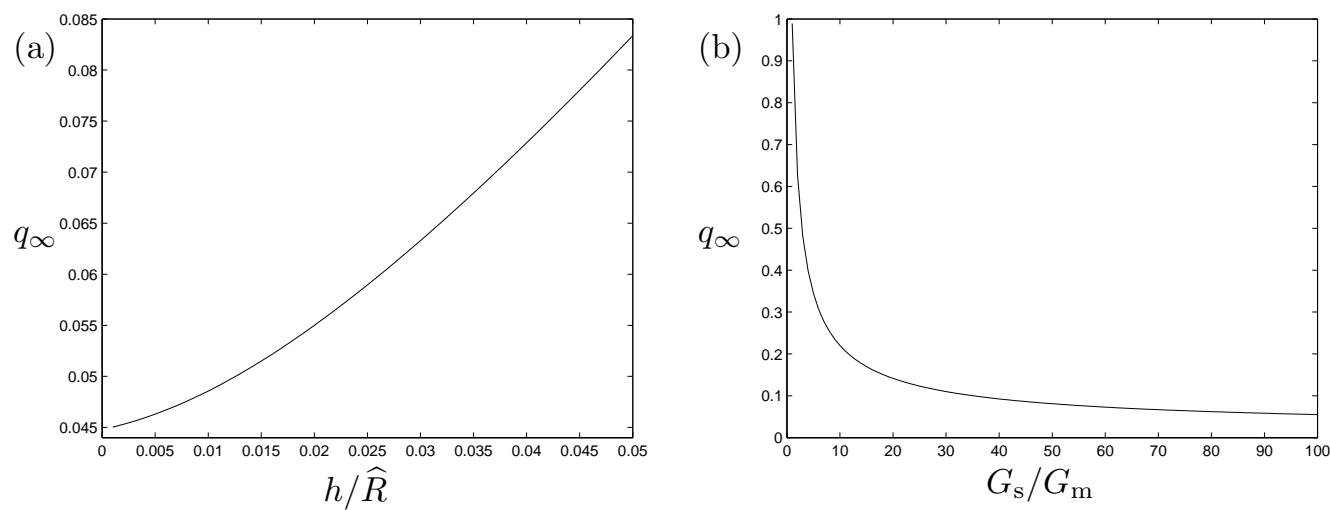

Fig. 4 Dependence of critical stress $q_{\infty}$ on parameters: (a) $h / \widehat{R}$, (b) $G_{\mathrm{s}} / G_{\mathrm{m}}$.

This buckling behaviour is reminiscent of that seen for beams on a nonlinear Winkler foundation (22) or, more significantly perhaps, for beams on an inhomogeneous foundation (23). In the latter case, buckling is localised at the point where the foundation is weakest; 

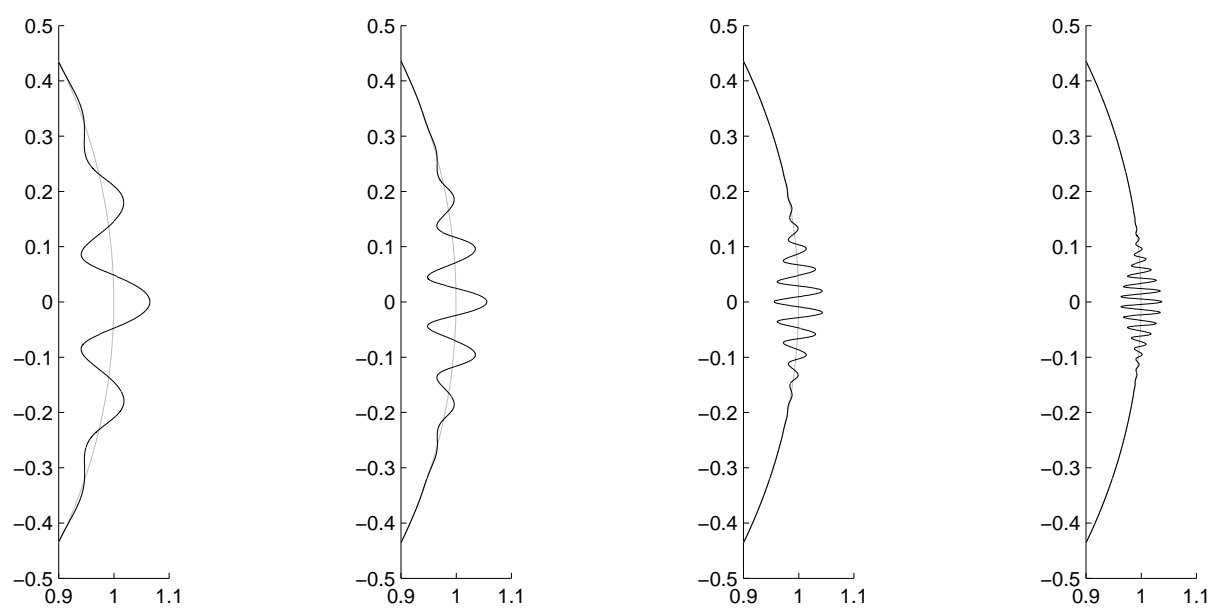

Fig. 5 Buckling patterns for $h / \widehat{R}=0.01,0.005, \quad 0.002,0.001$.

analogously, in the shell the localised buckling occurs where the pre-buckling compressive stress is greatest.

Finally, we note that by solving the eigenvalue problem above, we are also able to find the largest negative eigenvalue. This identifies the lowest critical tensile stress at infinity for which a buckled solution bifurcates from the equilibrium configuration (restricting buckling patterns to axisymmetric deformations). In both odd and even cases we find the critical stress to be $-q_{\infty}=0.1328$ for the material constants given previously. The corresponding buckling patterns are displayed in Figure 6.

(a)

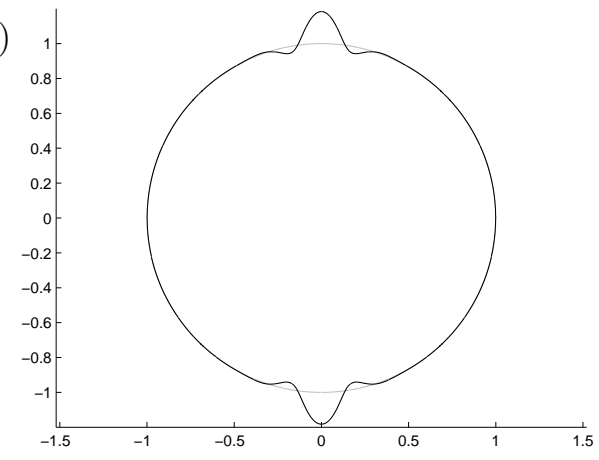

(b)

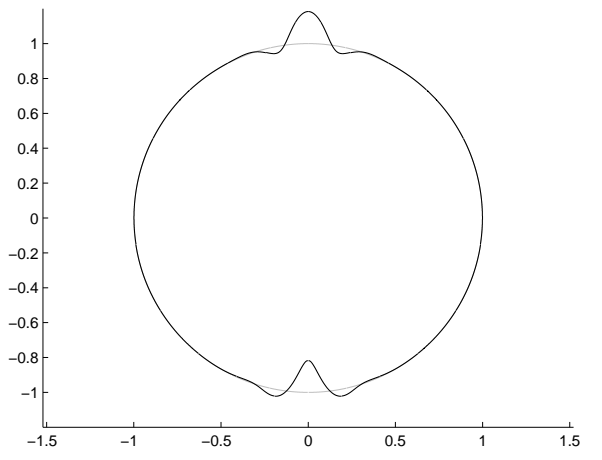

Fig. 6 Buckling patterns for the largest negative eigenvalue: (a) even, (b) odd. 


\subsection{Hydrostatic stress at infinity}

We will now calculate the lowest critical stress in the case where a hydrostatic stress is applied at infinity. Substituting (5.7) into the system (6.4), we deduce that $c_{n}=0$ for each $n$ since $p_{2}=0$. This means that $(6.4)$ becomes $\left(a_{n} q_{\infty}+b_{n}\right) \mathscr{U}_{n}=0$. Therefore each of the buckling modes are given from (5.1) by

$$
v_{R}=\mathscr{U}_{n} P_{n}(\mu), \quad v_{\theta}=\mathscr{V}_{n} P_{n}^{1}(\mu)=\mu_{n} \mathscr{U}_{n} P_{n}^{1}(\mu)
$$

for each $n$, with corresponding critical stress

$$
q_{\infty}=-\frac{b_{n}}{a_{n}}
$$

The constants $b_{n}$ in this expression are unchanged from (6.3), and substituting the relevant value of $p_{0}$ gives

$$
a_{n}=-\frac{3 \pi h\left(1-\nu_{\mathrm{m}}\right)\left(1+\nu_{\mathrm{s}}\right) G_{\mathrm{s}}}{\left(1-\nu_{\mathrm{s}}\right)\left(1+\nu_{\mathrm{m}}\right) G_{\mathrm{m}}} \frac{n(n+1)}{(2 n+1)} .
$$

We require the lowest critical stress at infinity, which involves finding the minimum value of (7.1) over $n=0,1,2, \ldots$. For the parameter values given previously, we obtain the lowest critical stress $q_{\infty}=0.08072$, for which $n=18$.

This result is to be compared with that of Fok and Allwright (9), who considered the buckling of an embedded shell with a hydrostatic stress field at infinity, but having introduced a simplifying assumption that the shell was inextensible, or that $\widehat{R} \nabla^{2} \psi+2 w=0$. This assumption gives $\mu_{n}=\frac{2}{n(n+1)}$, which is perhaps an oversimplification when compared to our result $(6.2)$.

In our notation, Fok and Allwright found that the critical stress at infinity for each mode $n$ satisfied

$$
\begin{gathered}
q_{\infty}=\frac{4 G_{\mathrm{s}}\left(1+\nu_{\mathrm{s}}\right)\left(1+\nu_{\mathrm{m}}\right)}{3\left(1-\nu_{\mathrm{m}}\right)}\left[1+\frac{G_{\mathrm{m}}\left(1-\nu_{\mathrm{s}}\right) \widehat{R}}{G_{\mathrm{s}}\left(1+\nu_{\mathrm{s}}\right) h}\right]\left\{\frac{2 h}{\widehat{R}(n-1)(n+2)\left(1+\nu_{\mathrm{s}}\right)}\right. \\
\left.+\frac{\left[n(n+1)-\left(1-\nu_{\mathrm{s}}\right)\right]}{12\left(1-\nu_{\mathrm{s}}^{2}\right)} \frac{h^{3}}{\widehat{R}^{3}}+\frac{G_{\mathrm{m}}\left[\left(2 n^{3}-n^{2}+3 n+2\right)-\nu_{\mathrm{m}}\left(2 n^{3}-3 n^{2}+5 n+2\right)\right]}{G_{\mathrm{s}}\left(1+\nu_{\mathrm{s}}\right)(n-1)^{2}(n+2)\left[3 n+2-2 \nu_{\mathrm{m}}(2 n+1)\right]}\right\}
\end{gathered}
$$

which, on minimising using our parameter values, gives the lowest critical stress as $q_{\infty}=0.4215$ when $n=18$. The value of $q_{\infty}$ compares quite badly with our result, indicating that the simplifying inextensibility assumption of the authors is not valid in our parameter regime (despite the fact that the order $n=18$ of the buckling pattern as calculated by Fok and Allwright agrees with the value arising from our theory). The results on letting the parameters $h / \widehat{R}$ and $G_{\mathrm{s}} / G_{\mathrm{m}}$ tend to zero are displayed in Figure 7 , and show a similar behaviour to the uniaxial case. The unbroken line corresponds to the results of our theory while the dashed line is the result of Fok and Allwright. Note that in the limit as either parameter tends to zero, the minimum $q_{\infty}$ according to (7.2) becomes asymptotically closer to the value given by our theory. 

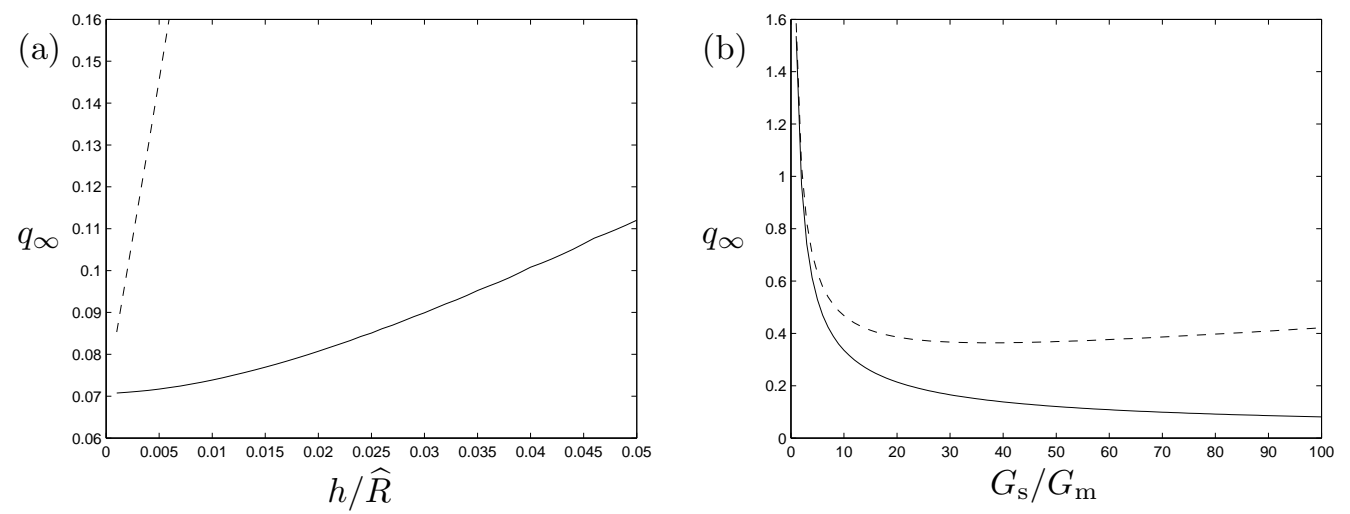

Fig. 7 Dependence of critical stress $q_{\infty}$ in the radial problem on parameters: (a) $h / \widehat{R}$, (b) $G_{\mathrm{s}} / G_{\mathrm{m}}$.

\section{Conclusions and discussion}

In analysing the buckling behaviour of an embedded shell, we have obtained a number of new results. The first is the observation that in uniaxial compression the shell buckles in a region around its equator, as opposed to near its poles. The reason for this is that buckling only occurs where the shell is in compression, which is the case in this equatorial region. The second result is an improved expression for the critical buckling stress when the stress field at infinity is purely hydrostatic, with confirmation that the result of Fok and Allwright are inaccurate when the thickness ratio $h / \widehat{R}$ is not exceedingly small. In addition we have analysed the behaviour of the shell undergoing uniaxial compression as two ratios tend to zero: the thickness ratio and the ratio of shear moduli $G_{\mathrm{S}} / G_{\mathrm{m}}$. In both limits, the buckling pattern becomes more oscillatory, which leads us to consider an analysis in these limits by WKB theory. Those results will appear elsewhere.

A few words should be said regarding the assumptions made during the analysis. The model chosen for the shell in order to analyse buckling was Koiter's shallow shell theory. This is the simplest nonlinear shell theory and the underlying assumption is that the wavelength of the buckling patterns is small compared to the radius of curvature of the shell. While this assumption seems to be confirmed a posteriori by the buckling patterns obtained, it may be worthwhile investigating more comprehensive nonlinear shell theories to verify that the observed buckling pattern justifies the use of the shallow shell assumption.

Secondly, we note that the results obtained are conditional on the buckling pattern being axisymmetric. While a verification of this conjecture depends on the calculation of the full nonaxisymmetric buckling problem, we believe that — at least for uniaxial compressive stress at infinity - the axisymmetric buckling pattern is likely to be the first to bifurcate from the equilibrium solution. One may justify this claim empirically by noting that the prebuckling stresses $\tau_{\theta \theta}$ and $\tau_{\phi \phi}$ are at their most negative at the equator, but the magnitude of $\tau_{\phi \phi}$ is considerably smaller than $\tau_{\theta \theta}$ for the chosen values of the Poisson ratios: specifically, in the limit $h / \widehat{R} \rightarrow 0,\left.\tau_{\phi \phi}\right|_{\mu=0}=\left.0.07 \tau_{\theta \theta}\right|_{\mu=0}$. Thus it is plausible that any stretching energy relieved by buckling in the $\phi$-direction would not compensate for the additional 
bending energy induced. However, a full nonaxisymmetric description of the buckling is required to prove or disprove this claim.

The main difficulty in implementing the nonaxisymmetric buckling problem is that the energy functional would become much more complicated; the quantities $w, \psi$ and $\chi$ would be linear combinations of surface spherical harmonics $Y_{n}^{m}(\theta, \phi)$ rather than Legendre polynomials. The relatively simple nature of our energy functional would thus not necessarily be reflected by one which includes variation in the $\phi$-direction. This will also be the case if we consider additional terms in the shell energy functional due to the use of a different shell theory.

Finally, we note that imperfections in the shell cause it to buckle at a lower critical stress than a pristine shell would. Koiter analysed this effect for a shell undergoing hydrostatic pressure by including an additional term in the energy functional, but to follow this approach for the embedded shell would be more difficult for the reasons outlined above.

\section{Acknowledgements}

GWJ would like to acknowledge the receipt of a CASE studentship, jointly funded by EPSRC and Dstl.

\section{References}

1. G. W. Jones, Static Elastic Properties of Composite Materials Containing Microspheres (D.Phil. thesis, University of Oxford 2007).

2. P. Seide, The stability under axial compression and lateral pressure of circularcylindrical shells with a soft elastic core, J. Aerosp. Sci., 29 (1962) 851-862.

3. M. J. Forrestal and G. Herrmann, Buckling of a long cylindrical shell surrounded by an elastic medium, Int. J. Solids Struct., 1 (1965) 297-309.

4. V. V. Vlasov, Stability of composite shells with an elastic core, Mech. Compos. Mater., 12 (1976) 499-502.

5. S.-L. Fok, Analysis of the buckling of long cylindrical shells embedded in an elastic medium using the energy method, J. Strain Anal., 37 (2002) 375-383.

6. O. Lourie, D. M. Cox, and H. D. Wagner, Buckling and collapse of embedded carbon nanotubes, Phys. Rev. Lett., 81 (1998) 1638-1641.

7. C. Q. Ru, Axially compressed buckling of a doublewalled carbon nanotube embedded in an elastic medium, J. Mech. Phys. Solids, 49 (2001) 1265-1279.

8. Y. F. Luo and J. G. Teng, Stability analysis of shells of revolution on nonlinear elastic foundations, Comput. Struct., 69 (1998) 499-511.

9. S.-L. Fok and D. J. Allwright, Buckling of a spherical shell embedded in an elastic medium loaded by a far-field hydrostatic pressure, J. Strain Anal., 36 (2001) 535-544.

10. W. T. Koiter, The nonlinear buckling problem of a complete spherical shell under uniform external pressure, Proc. Kon. Ned. Akad. B. Phys., 72 (1969) 40-123.

11. A. E. H. Love, A Treatise on the Mathematical Theory of Elasticity (Dover, New York 1944).

12. F. Zhaohua and R. D. Cook, Beam elements on two-parameter elastic foundations, $J$. Eng. Mech. ASCE, 109 (1983) 1390-1402.

13. F. I. Niordson, Shell Theory (North-Holland, Amsterdam 1985).

14. J. N. Goodier, Concentration of stress around spherical and cylindrical inclusions and flaws, Trans. ASME, 55 (1933) 39-44. 
15. S. H. Liu and E. B. Nauman, On the micromechanics of composites containing spherical inclusions, J. Mater. Sci., 25 (1990) 2071-2076.

16. M. Bilgen and M. F. Insana, Elastostatics of a spherical inclusion in homogeneous biological media, Phys. Med. Biol., 43 (1998) 1-20.

17. S. Mazzullo, Stress field around an $N$-layered spherical inclusion, In Proceedings of the European Mechanics Colloquium 214, 'Mechanical Behaviour of Composites and Laminates', Kupari, Yugoslavia, 1986, ed. W. A. Green and M. Mićunović (1987), pages $245-253$.

18. M. Rahman and T. Michelitsch, A general procedure for solving boundary-value problems of elastostatics for a spherical geometry based on Love's approach, $Q . J l$ Mech. Appl. Math., 60 (2007) 139-160.

19. H. L. Langhaar, Energy Methods in Applied Mechanics, (Wiley, New York 1962).

20. N. N. Lebedev, Special Functions and their Applications, (Dover, New York 1972).

21. A. I. Lur'e, Three-dimensional Problems of the Theory of Elasticity, (Interscience, New York 1964).

22. M. K. Wadee, G. W. Hunt, and A. I. M. Whiting, Asymptotic and Rayleigh-Ritz routes to localized buckling solutions in an elastic instability problem, Proc. R. Soc. Lon. Ser. A, 453 (1997) 2085-2107.

23. C. D. Coman, Inhomogeneities and localised buckling patterns, IMA J. Appl. Math., 71 (2006) 133-152. 